\title{
IUFOST2006/1022 Review of currently applied methodologies used for detection and typing of foodborne viruses
}

\author{
A. Rzezutka \\ National Veterinary Research Institute, Department of Food and Environmental Virology, Al. Partyzantow \\ 57, 24-100 Pulawy, Poland \\ arzez@piwet.pulawy.pl
}

In most foodborne outbreaks viruses are only indirectly implicated as a source of infection, due to difficulties in their detection. Taking into consideration that in many cases the food items can be contaminated by only a few virus particles and large sample size of the investigated food produce which needs to be tested, appropriate comprehensive procedures which will be able to combine all these issues have to be applied. Hitherto available virological methods (eg. cell culture infectivity assay, electron microscope) can not be fully used for analysis of food samples because they are laborious, time-consuming and have low sensitivity. Currently used methods for detection of foodborne viruses consist of two main stages: extraction and concentration of viruses from food samples and detection of viruses using molecular biology techniques (eg. reverse transcription - polymerase chain reaction). These methods are the most promising for detection of foodborne viruses, as they are fast, sensitive and can also allow typing of viruses. Despite the usefulness of such methods, there are differences in the sensitivity, specificity for different food matrices and detection limits of individual assays. There are several basic methods employing different procedures which can be used for virus extraction and detection in foodstuffs. Each of them has advantages and drawbacks. Some methods are insensitive, other do not have the specificity to allow identification of virus type or strain. This can result in variable efficiency, sensitivity of the method and can lead to the false negative results. The presentation deals with existing methodologies used for detection of hepatitis A virus and norovirus in fresh produce and food of animal origin, and discusses the requirements for their implementation in routine use. 